\title{
Photoconductivity of graphene devices induced by Terahertz radiation at various photon energies
}

\author{
M. Salman ${ }^{1,2}$, F. Gouider ${ }^{2,}{ }^{*}$, M. Friedemann ${ }^{4}$, H. Schmidt ${ }^{1,3}$, F. J. Ahlers ${ }^{4}$, M. \\ Göthlich $^{2}$, R. J. Haug ${ }^{1,3}$ and G. Nachtwei ${ }^{1,2}$
}

1. NTH School for Contacts in Nanosystems, Germany

2. Institut fuer Angewandte Physik, Technische Universitaet Braunschweig, D-38106 Braunschweig

3. Institut fuer Festkoerperphysik, Universitaet Hannover, D-30167 Hannover

4.Physikalisch Technische Bundesanstalt (PTB), Bundesallee 100, D-38116 Braunschweig

\begin{abstract}
The influence of a magnetic field on Landau levels (LLs) in graphene-based devices is described via the magnetooptical response induced by terahertz $(\mathrm{THz})$ radiation. For single-layer graphene, the resonance energies of the transitions between the on Landau levels (LLs) such as $\mathrm{L}_{1}, \mathrm{~L}_{2}$ and $\mathrm{L}_{3}$ fit quite well to the terahertz spectral range at low magnetic fields. Also, the calculations for the terahertz photoresponse (photoconductivity) in the presence of low magnetic fields, the reported calculations for the scattering rate of LLs, recent and our experimental results of photoresponse measurements yield that singlelayer graphene is suitable for the detection of terahertz radiation.
\end{abstract}

Keywords: terahertz, photoconductivity, graphene, Landau levels,

PACS: 78.67.De, 71.70.Di, 72.40.+w

\section{INTRODUCTION}

The increasing need for developing sources and detectors of the $\mathrm{THz}$ radiation has attracted keen interest in the $\mathrm{THz}$ regime to be sufficient for wider frames of applications. The detection of $\mathrm{THz}$ waves is complicated, compared to the generation of $\mathrm{THz}$ radiation. On the basis of non-resonant (bolometric) and resonance effects, various detectors for $\mathrm{THz}$ were developed. At low temperatures and relatively strong magnetic fields $(\mathrm{B} \approx 2 \mathrm{~T})$ quantum Hall $(\mathrm{QH})$ detectors which work on the basis of Landau quantization have been recently developed [1,2]. Here, we have presented a theoretical [3-7] and experimental studies for the magneto- optical response induced by terahertz $(\mathrm{THz})$ radiation in graphene. The linear dispersion relation of graphene leads [8,9] to the Landau levels (LLs) spectrum in which the transition energies are described as

$$
E_{n}= \pm v_{F} \sqrt{2 n|e B| \hbar / c}
$$

wehre \pm refers to electrons and holes and $v_{F}$ is Fermi velocity which plays the role of the speed of light in the Dirac equation. According to the equation above, the transition energies of allowed transitions between some of LLs such as $\mathrm{L}_{+1} \rightarrow \mathrm{L}_{+2}$ and as $\mathrm{L}_{+2} \rightarrow \mathrm{L}_{+3}$, yield the $\mathrm{THz}$ spectral range, at low magnetic fields. This is the first indication which can be used to argue that single-layer graphene is a potential detector for the $\mathrm{THz}$ radiation.

\section{THEORETICAL DESCRIPTION}

The magneto-optical response which arises due to the electronic transition between LLs is assumed to be proportional to the longitudinal conductivity [3-7]. The complex diagonal conductivity as given by Gusynin et al [7] can be briefly written (the full description is detailed in [3] and [7]) as:

$$
\sigma_{x x}(\omega, B) \propto \frac{B}{\omega} \sum_{m, n} \frac{f_{n}-f_{m}}{E_{n}-E_{m}-(\hbar \omega+i \Gamma)}
$$

where $M_{n}, f_{n}$ and $f_{m}$ are the energy, and occupation of the $n$th Landau level respectively and $\Gamma$ is the is the broadening of level due to the scattering rate. The Fermi energy $\mu$ and temperature dependence are included in the occupation functions $f_{n}$ and $f_{m}$.
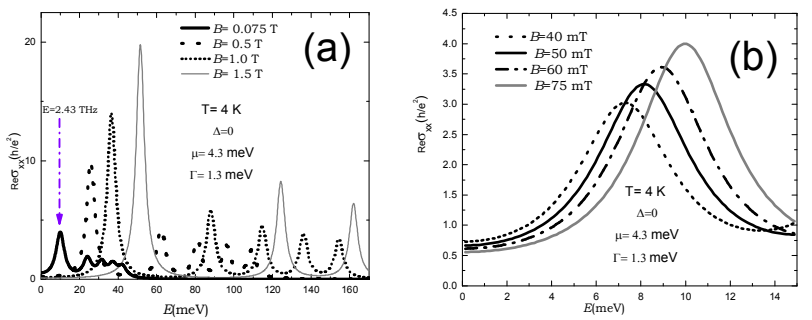

Figure 1. The real part of longitudinal conductivity $\left(\operatorname{Re} \sigma_{x x}\right)$ at different magnetic fields, $\mu=4.3 \mathrm{meV}, \Gamma=1.3 \mathrm{meV}$ and $\mathrm{T}=4 \mathrm{~K}$ plotted (a) versus wide range of infrared photon energy and (b) in the regime of THz energy.

In fig. 1, the scattering rate corresponds to $\Gamma=1.3 \mathrm{meV}$ as calculated at $1.7 \mathrm{~K}$ by Zhang et al [4]. 
Experimentally $[4,12]$, the Fermi energy $\mu$ can be tuned by a gate voltage. Thus, one can select a specific electronic transition with a specific signal strength depending on the index of the lower and upper levels.

\section{EXPERIMENTAL INVESTIGATIONS}

Our experimental investigations were achieved using a $p$-Ge laser emitting terahertz waves in the wavelength range between $120 \mu \mathrm{m}$ and $180 \mu \mathrm{m}$ [10]. Our laser pulses of a duration from 300 ns to some $\mu$ s with a maximum power of $1 \mathrm{~W}$.
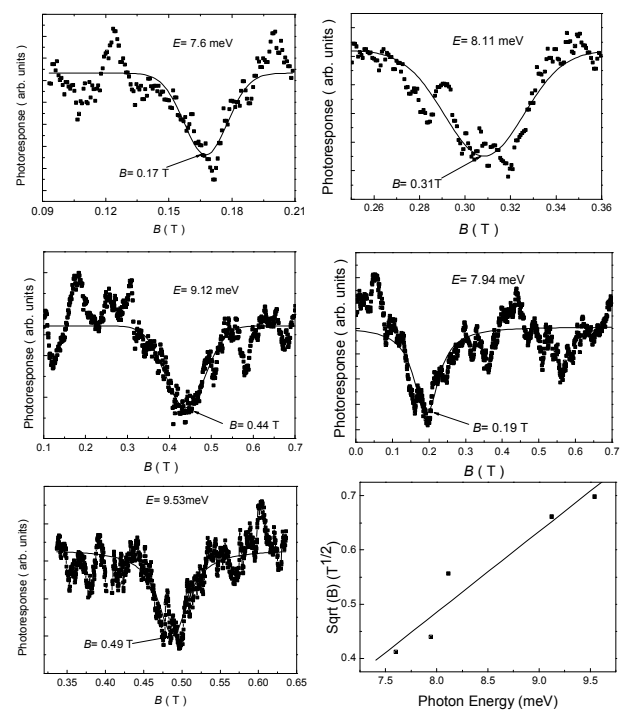

Figure 2. The observed photoconductivity peaks at low magnetic fields. The peak positions correspond to the resonance energies of the transitions between LLs. The plot of the field dependence of the photosignal energy demonstrates that the Landau levels separation is proportional to $\mathrm{B}^{1 / 2}$ as expected for single -layer graphene.

Our results are shown in fig. 2, in which the photoconductivity measurements were obtained at low temperature $(\mathrm{T} \sim 4 \mathrm{~K})$. In these measurements the magnetic field was swept at fixed $\mathrm{THz}$ photon energy. The measurements were achieved at different laser energies as given in fig. 2. Our sample is epitaxial graphene, in which a Corbino contact geometry was fabricated on SiC substrate (Si-face). On the other hand, a transmission measurement was obtained on the mentioned sample (without contact). Our result for the measurement exhibit a pronounced absorption peak around $\mathrm{B}=-0.788 \mathrm{~T}$ (fig. 3 ). The polarity dependence of our signal is due to the polarization of our $\mathrm{THz}$ laser. The polarization effect was not observed in the photoconductivity measurements. This could be due to the interaction between the electrons or holes in graphene and $\mathrm{THz}$ photons which is different in photoconductivity measurements from the one in transmission measurement.

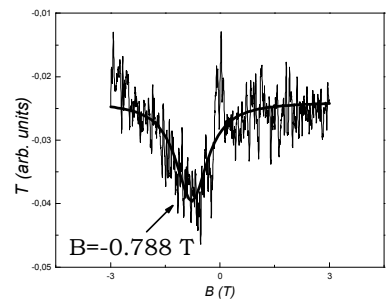

Figure 3. Transmission measurement of a Si-face graphene wafer exhibit a pronounced absorption peak around $\mathrm{B}=-0.788 \mathrm{~T}$.

\section{ACKNOWLEDGMENTS}

The progress of this work is supported by NTH School for Contacts in Nanosystems.

\section{REFERENCES}

1. F. Gouider, Yu.B. Vasilyev, M. Bugár, J. Könemann, P.D. Buckle, and G. Nachtwei Phys. Rev. B 81, 155304 (2010).

2. C. Stellmach, G. Vasile, A. Hirsch, R. Bonk, Yu.B. Vasilyev, G. Hein, C.R. Becker, and G. Nachtwei, Phys. Rev. B 76, 035341 (2007).

3. V. P. Gusynin, S. G. Sharapov, and J. P. Carbotte, Phys. Rev. Lett. 98, 157402 (2007).

4. Y. Zhang, Z. Jiang, J. P. Small, M. S. Purewal, Y.-W. Tan, M. Fazlollahi, J. D. Chudow, J. A. Jaszczak, H. L. Stoermer, and P. Kim, Phys. Rev. Lett. 96, 136806 (2006).

5. P. Neugebauer, M. Orlita, C. Faugeras, A.-L. Barra, and M. Potemski, Phys. Rev. Lett. 103, 136403 (2009).

6. N.M. R. Peres, F. Guinea, and A. H. Castro Neto, Phys. Rev. B 73, 125411 (2006).

7. M. Salman, F. Gouider, H. Schmidt, Yu. B. Vasilyev, R. J. Haug and G. Nachtwei. , Phys. Status Solidi C 8, No. 4, 1208-1210 (2011).

8. J. W. McClure, Phys. Rev. 104, 6661956.

9. F. D. M. Haldane, Phys. Rev. Lett. 61, 2015 (1988).

10. Yu. L. Ivanov and Yu. B. Vasilyev, Sov. Tech. Phys. Lett. 9, 264 (1983).

11. E. Gornik and A. A. Andronov (Eds.), Opt. Qauntum Electron. 23, 111 (1991).

12. K. S. Novoselov, A. K. Geim, S. V. Morozov, D. Jiang, Y. Zhang, S. V. Dubonos, I. V. Grigorieva, and A. A. Firsov, Science 306, 666 (2004). 
AIP Conference Proceedings is copyrighted by AIP Publishing LLC (AIP). Reuse of AIP content is subject to the terms at: http://scitation.aip.org/termsconditions. For more information, see http://publishing.aip.org/authors/rights-and-permissions. 